\title{
Back to The Future: How To Define Prognosis in MINOCA?
}

\author{
Luiz Eduardo Fonteles Ritt $^{1,2}{ }^{\oplus}$ and Mateus dos Santos Viana ${ }^{1,2}$ \\ Escola Bahiana de Medicina e Saúde Pública, 'Salvador, BA - Brazil \\ Instituto D'or de Pesquisa e Ensino (IDOR), Hospital Cárdio Pulmonar, ${ }^{2}$ Salvador, BA - Brazil \\ Editorial referring to the article: Acute Myocardial Infarction with Non-Obstructive Coronary Arteries - Stratifying the Risk of a "new" Clinical \\ Entity using an "Old" Tool
}

Myocardial Infarction with non-Obstructive Coronary Arteries (MINOCA) is the final diagnosis in $6-10 \%$ of all acute coronary syndromes (ACS). It is mostly present in female individuals. MINOCA was defined by the European Society of Cardiology (ESC) in 2017, and the Fourth Universal Definition of Myocardial Infarction (2018) further reinforced this concept. ${ }^{1,2}$

Dreyer et al., ${ }^{3}$ analyzing Medicare data in the United States, determined an incidence for MINOCA of 5.9\%. Lower rates of one-year MACE (1.7\% vs $27.6 \%$ ), mortality $(12.3 \%$ vs $16.7 \%)$ and re-hospitalization for ACS $(1.3 \%$ vs $6.1 \%$ ) were observed in MINOCA compared with ACS with coronary obstruction. Although the prognosis of MINOCA seems to be better than conventional ACS, a better understanding of which conditions are associated with better or worse prognosis within the MINOCA spectrum is needed.

In this issue of the International Journal of Cardiovascular Sciences, Carvalho et al., ${ }^{4}$ applied the GRACE score in 56 patients with suspicion of MINOCA and found an excellent discriminatory accuracy for mortality (AUC 0.907; 95\%CI 0.812-1.000; $\mathrm{p}=0.019$ ) and occurrence of MACE (AUC 0.790; 95\%CI 0.632-0.948; $\mathrm{p}<0.05)$. They also showed a higher event rate for each GRACE score cut off $(<114,114-137$ and $>=138)$.

Yin et al., ${ }^{5}$ recently published data regarding the use of the GRACE score in a retrospective cohort of MINOCA patients in Shangai and found a worse

\section{Keywords}

Cardiovascular Diseases; Myocardial Infarction; Acute Coronary Syndrome; Coronary Artery Disease; Coronary Angiography; Magnetic Resonance/diagnostic imaging. prognosis for patients with higher GRACE score (> 140 points) compared with those at low/intermediate risk defined by the score. The occurrence of MACE was higher in those with high GRACE score $(21.9 \%$ vs $10.2 \% ; \mathrm{p}<0.01$ ), and this difference was driven particularly by the component cardiac mortality $(7.5 \%$ vs $1.4 \% ; \mathrm{p}<0.05)$. Also, they found an AUC of 0.710 (95\% CI 0.625-0.796, p < 0.001).

Results of both studies reinforce the use of the GRACE score for MINOCA in daily practice. As the GRACE score may help in stratifying patients before coronary angiography, it has already been used in most centers for all patients who present with suspected ACS, including MINOCA, as the final diagnosis of MINOCA will be made only after the coronary profile is established. Thus, in some cases, the score prevents the patient from being submitted to unnecessary tests.

But what explains the prognostic value of GRACE risk score in MINOCA?

This question should be answered from two perspectives. The first regards the pathophysiological mechanism of MINOCA. In a series of MINOCA patients, ${ }^{6}$ cardiac magnetic resonance and optical coherence tomography (OCT) revealed an ischemic nature of the disease in $63.8 \%$ of patients. In this scenario the performance of GRACE score would be kept. In the study by Carvalho et al., ${ }^{4}$ cardiac magnetic resonance was performed in only $21.4 \%$ of the patients and OCT was not performed, and the underlying mechanism of the disease was established in only $17.9 \%$ of the cases.

Second, despite being a poor predictor of anatomical severity $^{7}$, the GRACE score will always be a good predictor of insult severity, regardless of anatomy.

Mailing Address: Luiz Eduardo Fonteles Ritt 
The individual components of the GRACE score provide prognostic information that are not specific of ACS. In fact, age, heart rate, systolic blood pressure, the Killip class (presence of pulmonary edema or cardiogenic shock), troponin and even ST changes play a general role in the prognosis and accurately predict mortality; the outcome depends more on patients' susceptibility to the stress event than on atherosclerotic burden per se. For example, the GRACE score was also shown to provide good prognostic information

\section{References}

1. Thygesen K, Alpert JS, Jaffe AS, Chaitman BR, Bax JJ, Morrow DA, et al. ESC Scientific Document Group, Fourth universal definition of myocardial infarction (2018), Eur Heart J.2019;40(3):237-69. https:// doi.org/10.1093/eurheartj/ehy462

2. Agewall S, Beltrame JF, Reynolds HR, Niessner A, Rosano G, Caforio AL,et al. Per Tornvall, on behalf of the WG on Cardiovascular Pharmacotherapy, ESC working group position paper on myocardial infarction with nonobstructive coronary arteries. Eur Heart J.2017;38(3):143-53. https://doi. org/10.1093/eurheartj/ehw149

3. Dreyer RP, Tavella R, Curtis JP, Pauspathy S Y, Messenger J, Rumsfeld JS,et al. Myocardial infarction with non-obstructive coronary arteries as compared with myocardial infarction and obstructive coronary disease: outcomes in a Medicare population, Eur Heart J.2020;41(7):870-8. https:// doi.org/10.1093/eurheartj/ehz403

4. Carvalho P, Caçoilo M, Afreixo V, Bastos JM, Ferraz L, Vieira M, et al. Acute Myocardial Infarction with Non-Obstructive Coronary Arteries Stratifying the Risk of a "new" Clinical Entity using an "Old" Tool. Int J Cardiovasc Sci. 2021;

5. Yin G, Abdu FA, Liu L, Xu S, Luo Y, Lv X, et al.Prognostic Value of GRACE Risk Scores in Patients With Non-ST-Elevation Myocardial Infarction With Non-obstructive Coronary Arteries. Front Cardiovasc Med.2021;8:582246. doi:10.3389/fcvm.2021.582246

6. Reynolds HR, Maehara A, Kwong RY, Sedlak T, Saw J, Smilowitz NR, et al. Coronary Optical Coherence Tomography and in Takotsubo cardiomyopathy ${ }^{8,9}$ and pulmonary embolism. ${ }^{10}$

Finally, in a significant proportion of cases, MINOCA may not be indissociable from ACS and may be interpreted as a continuum in atherosclerotic disease. ${ }^{11}$ In this case, patients should be advised and counseled appropriately, beyond the aspects of coronary heart disease. New studies evaluating the relation of other classical prognostic factors to atherosclerosis in MINOCA are warranted.

Cardiac Magnetic Resonance Imaging to Determine Underlying Causes of Myocardial Infarction With Nonobstructive Coronary Arteries in Women. Circulation. 2021;143(7):624-40. doi: 10.1161/ CIRCULATIONAHA.120.052008.

7. Barbosa CE, Viana M, Brito M, Sabino M, Garcia G, Maraux M, et al. Accuracy of the GRACE and TIMI scores in predicting the angiographic severity of acute coronary syndrome. Arq Bras Cardiol. 2012 Sep;99(3):818-24. doi: 10.1590/s0066-782x2012005000080.

8. Zalewska-Adamiec M, Kuzma L, Dobrzycki S, BachorzewskaGajewska H. The GRACE Scale in the Prognosis of Patients with Takotsubo Syndrome. J Interv Cardiol. 2020 Apr 24;2020:4340930. doi: $10.1155 / 2020 / 4340930$

9. Scudiero F, Arcari L, Cacciotti L, De Vito E, Marcucci R, Passaseo I, et al. Prognostic relevance of GRACE risk score in Takotsubo syndrome. Eur Heart J Acute Cardiovasc Care. 2020 Oct;9(7):721-8. doi: $10.1177 / 2048872619882363$.

10. Paiva LV, Providencia RC, Barra SN, Faustino AC, Botelho AM, Marques AL. Cardiovascular risk assessment of pulmonary embolism with the GRACE risk score. Am J Cardiol. 2013 Feb 1;111(3):425-31. doi: 10.1016/j. amjcard.2012.10.020.

11. Ciliberti G, Guerra F, Coiro S, Capucci A. Is there an 'atherosclerotic continuum' from angina with unobstructed coronary arteries to MINOCA?, Eur Heart J.2019;40(24):1987. https://doi.org/10.1093/ eurheartj/ehy914 